\title{
Evaluation Of Tooth Enamel Content Exposed To Bleaching Gel
}

\author{
J. Silveira*, S. Pessanha**, J. Godinho*, M. Albergaria*, M.M. Lopes***, A. Mata* and M.L. Carvalho** \\ * Grupo de investigação em Bioquímica e Biologia Oral, Unidade de Investigação em Ciências Orais e \\ Biomédicas da Faculdade de Medicina Dentária da Universidade de Lisboa, Cidade Universitária 1649-003 \\ Lisboa, Portugal \\ ** Centro de Física Atómica da Universidade de Lisboa, Av. Professor Gama Pinto 2 1649-003 Lisboa, \\ Portugal \\ *** Departamento de Biomateriais, Unidade de Investigação em Ciências Orais e Biomédicas da Faculdade \\ de Medicina Dentária da Universidade de Lisboa, Cidade Universitária 1649-003 Lisboa, Portugal
}

Email: joaosilveiramd@gmail.com

Tooth bleaching is an effective, conservative and relatively inexpensive treatment for correction of tooth discoloration $[1,2,3,4]$. However, there is some controversy regarding the influence of hydrogen peroxide (HP) on enamel surface morphology and oral tissues biology [3,4]. Currently there are two main techniques used for tooth bleaching: in-office, using high HP concentrations (6-40\%) and at-home prescribed bleaching commonly using carbamide peroxide (CP) with concentrations ranging from $10 \%$ to $20 \%$.

This in vitro study aimed to assess the effects of tooth bleaching products on enamel element content and micromorphology, using Micro X-ray fluorescence ( $\mu$-XRF) and Scanning Electron Microscopy (SEM).

Healthy teeth, extracted for periodontal or orthodontic reasons, were selected and preserved in a $0.5 \%(\mathrm{w} / \mathrm{w})$ chloramine solution for no longer than 6 months. After confirming that there were no irregularities in the vestibular surfaces of the teeth, cuts were made in order to obtain samples with approximately $8 \mathrm{~mm} \times 2 \mathrm{~mm}$. Samples were divided in two groups: Group 1 - OPL Boost or Group 2 - OPL PF and treated with the bleaching products (Opalescence Boost, representing the in-office technique and containing 40\% of HP; and Opalescence PF 10\%, representing the at-home and gold standard technique, containing $10 \%$ of $\mathrm{CP}$ ). Products were applied accordingly to manufacturer instructions (Ultradent, USA). For SEM analysis, samples were incubated in artificial saliva until pre-determined periods. Digital photographs of three areas (A, B and C) were obtained at different magnifications. For $\mu$-XRF analysis, after bleaching element content of each sample was measured before and immediately after treatment. The quantitative analysis was carried out with appropriated software package and statistical treatment, paired-sample T-test, performed by SPSS V.21 (IBM, USA) and presented as \% (w/w) or ppm (w/w).

The results of this study show a decrease in enamel element content of strontium in Group $1(\mathrm{P}<0.05)$. Reductions of copper and zinc content have been registered in both groups $(\mathrm{P}<0.05)$. No significant changes were observed for major elements present (calcium and phosphorus) ( $P>0.05$ ). These results of $\mu$-XRF analysis suggest a decrease of trace-element, more commonly present in enamel surface, by the effect of bleaching procedure. However the irregular topographic distribution and so an measurement bias of such elements should not be excluded.

Enamel micromorphological changes were recorded immediately after application when compared with control samples. However, these changes were not recorded after incubation in artificial saliva for a minimum period of 14 days, suggesting a remineralizing effect of saliva following both bleaching procedures [5].

Within the limitations of this in vitro study, it can be concluded that the application of booth tooth bleaching gels were able to reduce enamel mineral content of trace elements. 
[1]Cardoso, P. C. et al., J Am Dent Assoc,, 141, 1213-20, 2010.

[2]Fugaro, J. O. et al., Oper Dent, 29, 363-8, 2004.

[3]Joiner A. J Dent., 35, 889-96, 2007.

[4]Maia E. et al., J Dent., 36, 2-7, 2008.

[5]Amerogen, A.V. and Veerman, E.C., Oral Dis, 8, 12-22.
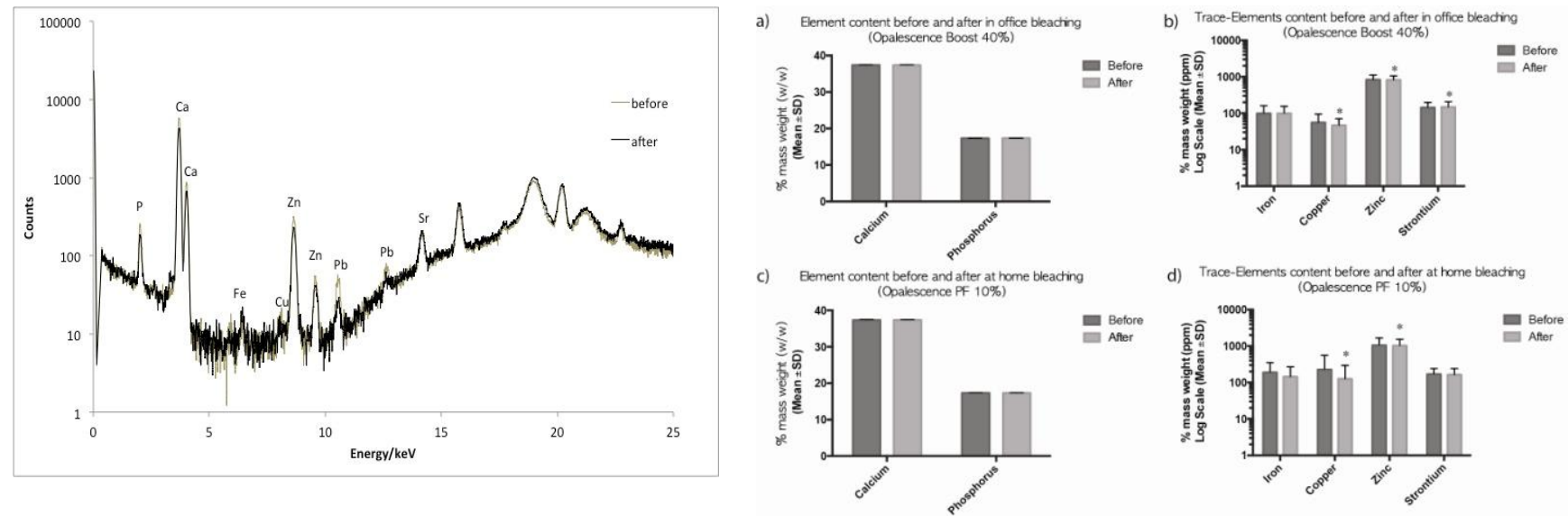

Figure 1 - Typical spectra obtained before and after tooth bleaching procedure

Figure 2 - Graphs showing element [a) and c)] and trace-element [b) and d)] before and after tooth bleaching procedure in-office (Opalescence Boost) and at-home (Opalescence PF 10\%). * statistical differences, $\mathrm{P}<0.05$

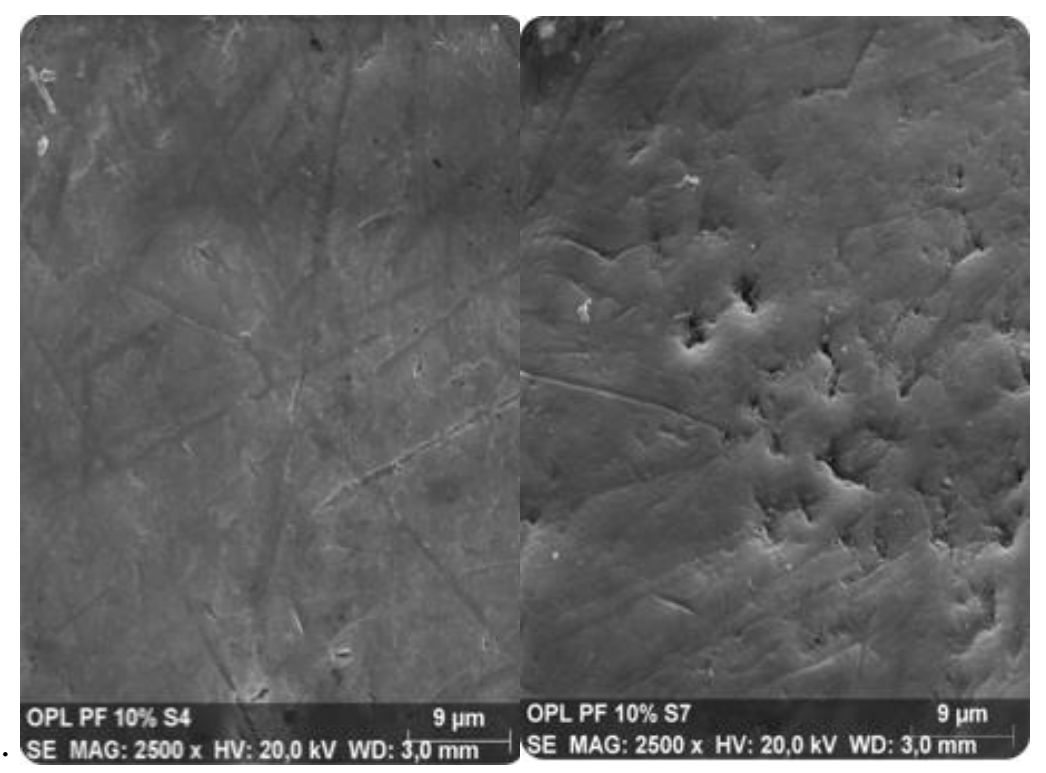

Figure 3 - Typical SEM images of enamel rnicro morphologic surface for control [a)] and Group 2 [b)] (x2500). 